\title{
GECEM: A Portal-Based Grid Application for Computational Electromagnetics
}

\author{
Maria Lin and David W. Walker* \\ School of Computer Science, Cardiff University, 5 The Parade, Roath, Cardiff CF24 3AA, U.K.
}

\begin{abstract}
The Grid-Enabled Computational Electromagnetics (GECEM) portal is a problem-solving environment that uses grid technologies to support scientists in accessing distributed resources for the solution of computational electromagnetics (CEM) problems. These resources include input files specifying the system geometry, and proprietary software and hardware for mesh generation and CEM simulation. Through interacting with a web-based grid portal, a user can access these resources, submit jobs, monitor and execute distributed grid applications, and collaboratively visualize the results of the CEM simulation. Thus, the portal allows users to use Grid infrastructure to share resources among geographically distributed partners in order to execute large-scale computational electromagnetics simulations, and to collaboratively analyze and visualize the results.

This paper describes a secure, web-based portal, built on the GridSphere framework, composed of a number of JSR-168 compliant portlets that deploy services at the back end for discovery and management of distributed resources, and for invoking services for mesh generation and CEM simulation. The paper also describes how security is achieved through the Grid Security Infrastructure and single sign-on.
\end{abstract}

Key words: Computational electromagnetics, portals, portlets, Web services

\section{Introduction}

Grid-based portals are an increasingly popular way of allowing computational scientists to perform collaborative research and share geographically-dispersed resources [1]. Scientific simulations are often both data- and compute-intensive, and may involve heterogeneous resources running under different operating environments. Such simulations

\footnotetext{
* Corresponding author.

Email addresses: linsl@adf.bham.ac.uk (Maria Lin), david@cs.cf.ac.uk (David W. Walker).
} 
typically involve complex interactions between a number of systems. The Open Grid Service Architecture (OGSA) [2] describes an infrastructure which is suitable for collaborative resource sharing via the Grid. However, the installation and use of this grid infrastructure is generally complex and difficult for all but trained experts. Grid middleware provides mechanisms for performing low-level grid tasks such as transferring files, executing processes and monitoring process output. However, in general, scientific end users do not have the expertise, or the desire, to directly perform these low-level tasks themselves. Web-based grid portals provide an integrated user interface that not only provides a single point of access to heterogeneous resources, but also hides the complexities associated with their use. Scientists can work in terms of application domain concepts, and can concentrate on their research without worrying about the complexities of using the Grid.

Computational electromagnetics (CEM) is of increasing importance to the civil and defence sectors, and is central to important problems such as predicting the electromagnetic compatibility between complex electronic systems, and the response of systems to lightning strikes and electromagnetic pulses. Large-scale CEM simulations are computationally intensive, and can involve access to resources that are intrinsically distributed. For example, in the case of an "extended enterprise" in which multiple partners from industry and academia are cooperating to design and build a complex system that requires CEM simulations, the geometry of a component may be created at one location, a mesh conforming to this geometry may be generated at a second location, and a CEM simulation based on the mesh may be performed at a third location. Finally, the output from the simulation may be analyzed and visualized at one or more other locations. Thus, CEM simulations are suitable candidates for use of the Grid.

This paper describes the Grid-Enabled Computational Electromagnetics (GECEM) project, focusing in particular on how it makes use of grid technologies to support collaborative research through the design and implementation of a portal based on portlets and services. The portal enables remote mesh generation, and the migration of the CEM solver code to be executed at a remote location. The portal supports collaborative simulation and access to distributed resources among the different project partners in a user-friendly approach. To this end, the GECEM portal has been designed to support the generation of computational meshes using meshing services at the University of Wales, Swansea, the solution of a CEM problem on this mesh on a supercomputer at the Singapore Institute of High Performance Computing, and the collaborative visualization of the results by participants at Cardiff University and BAE Systems in Bristol [3].

The remainder of this paper is organized as follows. Section 2 discusses the design of the GECEM portal, and describes the design requirements and key features of the portal. Section 3 presents the architecture of the GECEM portal, and discusses the portal and portlet technologies on which it is based. Implementation aspects are considered in Section 4. Related work is discussed in Section 5, and conclusions and directions for future work are presented in Section 6 .

\section{Design of the GECEM Portal}

The GECEM project involved both industrial and academic partners, the former being BAE Systems and Hewlett-Packard, and the latter Cardiff University and the University 
of Wales, Swansea. The other project partner was the Singapore Institute of High Performance Computing, which is an industry-focused research organization. The design of the GECEM portal was a collaborative and iterative process involving project partners that sought to match end user requirements to technical solutions within the budget and two-year time span of the project. This section discusses the most important outcomes of this design process.

\subsection{Requirements}

The GECEM portal must satisfy requirements in the following areas:

User Access. The portal should provide users with a single point of access, without the need to login to multiple different machines. The portal should also support simultaneous access by multiple users.

Job Workflow. A GECEM workflow takes as input a geometry file, and uses this to evaluate conforming surface and volume meshes by executing two distinct codes. A third executable takes the surface and volume mesh files as input and performs a CEM simulation. A GECEM job consists of running all or part of such a workflow, and the portal should support the configuration, submission, and monitoring of GECEM jobs.

Visualization. Once a CEM simulation is complete, users should be able to collaboratively visualize the result within the portal.

Job Management. Users should be able to submit multiple jobs at the same time. Users should be able to log out of the portal and the job should still continue to run. Subsequently users should be able to login to the portal to check on the progress of their jobs.

Legacy Code. The portal should be able to transparently access legacy codes to perform the numerical tasks in a GECEM workflow.

CEM simulation migration. High quality CEM solver codes often represent significant investments of time and money, and may give a business a competitive edge that would be lost if their codes were accessible to others. Thus, the owner of such a CEM solver code may not want to install it permanently on multiple computers outside of their organization. The portal should support the secure migration of the CEM simulation executable code to a selected target machine, together with any necessary input data sets. The code should then execute, its output stored in a user-specified location, and the code on the target machine deleted, along with any related data sets.

Service Discovery. The services accessible from the portal for computation and visualization must be registered with a resource registry, and be discoverable from within the portal.

Resource Selection. Users should be able to browse multiple machines to select input files, and be able to specify where the output files are stored. When the service discovery mechanism discovers multiple services capable of performing the same task, users should be able to select which one they wish to use.

Security. The portal must allow users single sign-on access to resources through their security credential, such as a UK e-Science certificate. Single sign-on refers to the ability of a user to perform a single action of authentication (such as entering a password) to access the distributed resources that he or she is authorized to use. In addition, delegation of credentials must be supported so that tasks such as file access and job 
execution can be preformed on a user's behalf, and any files generated are owned by the user. Delegation is a mechanism whereby a user or service can delegate a subset of their access rights to another service.

\subsection{Features of the GECEM Portal}

The GECEM portal satisfies all the requirements listed in Section 2.1. The legacy codes for surface and volume mesh generation are exposed as Grid services, and the CEM migration process is also controlled by a Grid service. Of course, the execution of the CEM simulation code on the remote host is not entirely secure, but the approach adopted reduces the risk of an organization's valuable software being accessed by unauthorized parties. The mesh generation and solver migration services are registered in a Universal Description, Discovery and Integration (UDDI) [4] registry. The portal can discover these services dynamically and invoke selected services to generate surface and volume meshes, and to migrate the solver executable code to a remote location.

The GECEM portal is designed to support a pipeline style of workflow in which the nodes correspond to the surface mesh, volume mesh and CEM migration services. The surface mesh service takes as its main input a file specifying the geometry of the problem to be simulated. Geometry files are assumed to be generated outside of the portal, and are stored in a geometry archive that can be browsed and managed within the portal. The surface and volume mesh services output surface and volume meshes, respectively, and these are stored in their own archives. Finally, the CEM solver service takes mesh files as input and generates the solution, which is stored in the solution archive.

The GECEM portal allows a user to set up a job as a sequence of one or more services, with specified input and output files. Where multiple equivalent services are available for a particular task, perhaps employing different algorithms or numerical methods, the user can select from these. Having decided which services to use, the input files for the job are selected from the appropriate archive. In the full three-stage workflow an input geometry file is selected from the geometry archive as input for the surface mesh service. The output from this is passed to the volume mesh service, which in turn passes its output to the CEM migration service. The output from this latter service is stored in the solution archive. By default all intermediate outputs from the surface and volume mesh services are archived, so a job could, for example, consist of just the CEM migration service, with the input being selected from the surface and volume mesh archives. A three-stage GECEM workflow is shown in Fig. 1, in which the dashed arrows into the archives show how data sets can be archived. The dashed arrows leading from the archives shows how one- and two-stage workflows can be set up using archived input data sets.

Another important aspect of the GECEM portal is its support for collaborative visualization and analysis. Multiple distributed users can collaboratively visualize and navigate data sets in the portal's archives. Two modes of collaborative visualization are supported: one in which all participants see the data set from the same point of view as a designated "leader", and the other in which the participants navigate the data set independently. Support for collaborative visualization is discussed further in Section 3.2.

Security is achieved through use of the Grid Security Infrastructure (GSI), and the user's credential is delegated from the client to the service. This is discussed further in Section 4. 


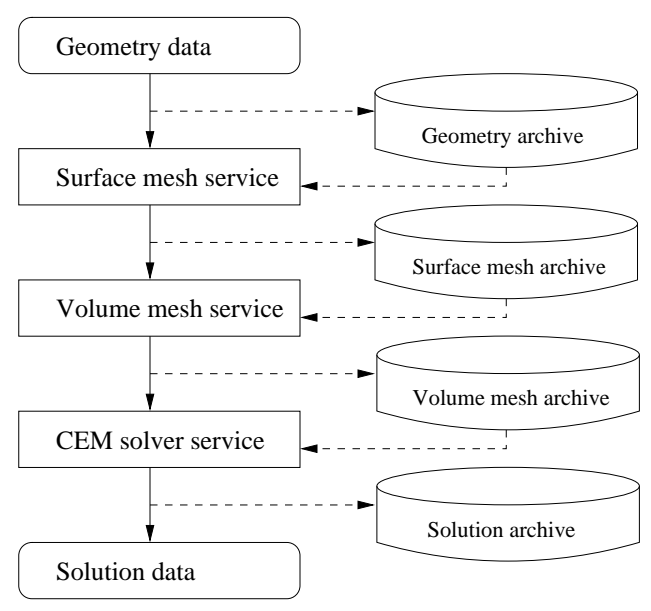

Fig. 1. A three-stage GECEM workflow

\section{Architecture of the GECEM Portal}

The GECEM portal is a problem-solving environment composed of a collection of JSR168-compliant portlets ${ }^{1}$ and services for mesh generation and CEM simulation. A portlet is a pluggable user interface component used within the context of a portal framework. From a user's point of view a portlet is a window in a portal that provides a specific service or function. A portlet processes requests and generates dynamic content, and the content of multiple portlets are typically aggregated together to form a portal web page. A portlet's life cycle is managed by a portlet container. Portlet standards, such as JSR168 and Web Services for Remote Portlets (WSRP), are helping to make portlet-based portals the most common way of presenting aggregated web content to consumers [5]. The GECEM portal provides the main interface through which services are accessed. The portal supports the composition of applications from service-based components, the execution and monitoring of such applications on remote resources, and collaborative visualisation and exploration of the application results.

Two key decisions on the design of the portal were made early in the project. The first was to use the publicly-available open-source GridSphere portal framework [6] as the container of the GECEM portal. The GridSphere framework consists of a portal server and a number of core portlets. GridSphere provides a container to manage the portlets, and allows users to login to the portal and customize it. The second design decision was to base the GECEM services on Globus Toolkit 3.2, as this was the most recent version of the toolkit available early in the project. GT4 was not available until close to the end of the project, and it was decided not to migrate the services to this version of the toolkit.

In addition to the GridSphere portal framework, GECEM portal consists of three other main components: the GridPortlets web application [7], the GECEM Portlets, and the RAVE Portlet. The GridPortlets web application, developed by the same research team that developed GridSphere, supports the definition of resources through a Resource Registry, and a Credential Management Service for using grid services. GridPortlets also

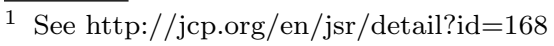


provides a set of portlets for tasks such as credential retrieval, resource browsing, file browsing, file transfer, and job submission.The GECEM Portlets are the main components which allow users to select files, discover the meshing and CEM migration services, submit jobs for remote execution, and monitor jobs. The RAVE portlet allows users to collaboratively visualize the outputs generated from jobs. Figure 2 presents the GECEM portal architecture.

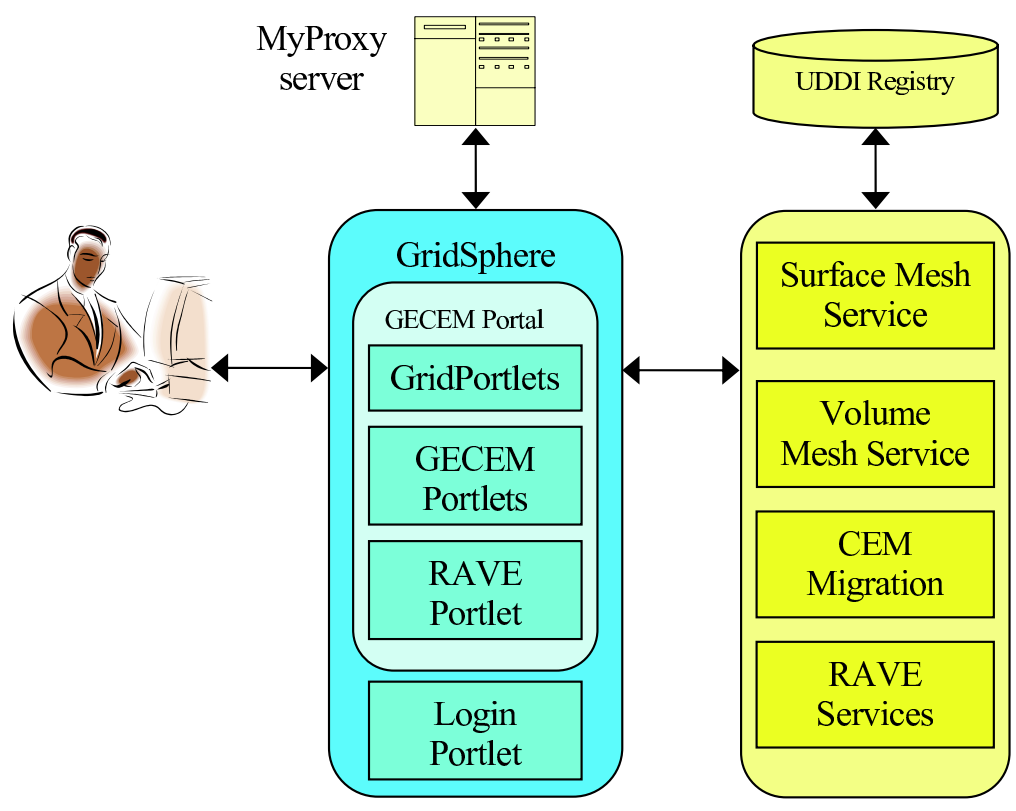

Fig. 2. The main components of the GECEM portal architecture.

\subsection{GECEM Portlets}

The GECEM portlets are built on top of GridSphere, and that make use of the features provided by the GridPortlets web application to support Grid applications. To perform a task in the GECEM workflow (see Fig. 1) requires the user to make use of GECEM portlets to do the following actions:

(i) Select the input files and the desired directory for the output of the task.

(ii) Search the UDDI registry for the Grid Service Handle (GSH) of the factory service of all services that can perform the task.

(iii) Select one of the discovered services.

(iv) Invoke the service.

The GECEM File Selection Portlet is based on the GridPortlets File Browser Portlet. The File Selection Portlet allows the user to select an input file by first choosing the machine where the file is stored, and then browsing to the file location on that machine. The list of machines that the user chooses from is populated from a GridPortlets configuration file that indicates which machines support GridFTP. GridFTP is used to transfer input files from where they are stored to the service host. The File Selection Portlet also allows the user to select, in a similar way, the directory where output from a service is 
stored. A File Parameter Portlet is also provided that shows, in URI format, the names of the files and directories selected so that users can easily check if they have made the correct selections, and make corrections if necessary.

Service discovery and selection is performed in the GECEM UDDI Portlet. To allow a service to be discovered, the Grid Service Handle (GSH) of the factory service of the services to be used by GECEM are published to the UDDI registry at the Welsh e-Science Centre (WeSC). The UDDI portlet is set by default to search the WeSC UDDI registry. The UDDI Portlet allows the user to search the UDDI registry for information on the location of the available mesh generation and CEM solver migration services, thereby allowing them to be discovered dynamically. The UDDI Portlet presents a list of services discovered, and the user then selects the required service.

The GECEM Service Invocation Portlet uses the input and output file locations from the File Selection Portlet, and the GSH from the UDDI Portlet to invoke the corresponding remote grid service (at The University of Wales, Swansea). The portal is set up to execute the CEM simulation on an 8-processor SGI machine at Cardiff University. When the user invokes the CEM Solver Migration Service it transfers the inputs files and the solver executable code through GridFTP to the Cardiff machine, and when the job is completed, the files are transferred back to a user-specified location.

Since the actions enumerated above must be performed for each stage in a GECEM workflow, there are service-specific GECEM portlets for surface mesh generation, volume mesh generation, and CEM solver migration that make use of the generic GECEM portlets described in the preceding paragraphs for file selection, service discovery and selection, and service invocation.

A further set of GECEM portlets is provided to display to users information about jobs that they have submitted. The Job Submission Portlet, which makes use of the GridPortlets Job Submission Service, allows users to view the input and output file details, and the status of jobs. The Progress Portlet displays a log of the events that occur during service invocation, such as the transfer of files. Finally, for the CEM simulation, the Result Portlet shows the percentage of the task completed, based on a count of loop executions in the code.

\subsection{RAVE Portlet}

The Resource-Aware Visualization Environment (RAVE) was used to provide a collaborative visualization capability within the GECEM portal. RAVE is a collaborative visualization environment that scales across visualization platforms, ranging from large immersive devices all the way down to hand-held PDAs [8]. RAVE is based on web service technologies, and provides for distributed rendering on remote machines. The data to be rendered may reside on one machine, the rendering may be done on one or more other machines, and the rendered image may be displayed on yet another machine.

A RAVE Portlet was developed and integrated into the GECEM portal. The RAVE Portlet allows the user to select resources to provide the RAVE functionality. These are discovered dynamically using a UDDI service. The user can also initiate a collaborative visualization session, which users at other locations can also join, and select data sets to render. The data set will then be displayed in the GECEM portal on the local client, and on any other machines that have joined the collaborative session. Users can then navigate, 
and interact with, the data set. Users are represented graphically in the visualization by an avatar, which can be seen by other users in the collaborative session. An avatar is shown in Fig. 3, which is taken from a collaborative session visualizing a sphere. The figure shows the viewpoint of one of the people involved in the collaborative session. The avatar of another person in the session can be seen represented by the characters "DWW". The nearby 3-D arrow indicates the direction in which DWW is looking. The characters are shown back-to-front because the avatar represents someone who is between the sphere and the viewpoint. If the DWW avatar were on the far side of the sphere (and not obscured by it) the characters would be seen the right way round. The lower part of the figure shows part of the control panel for navigating and manipulating the visualized data.

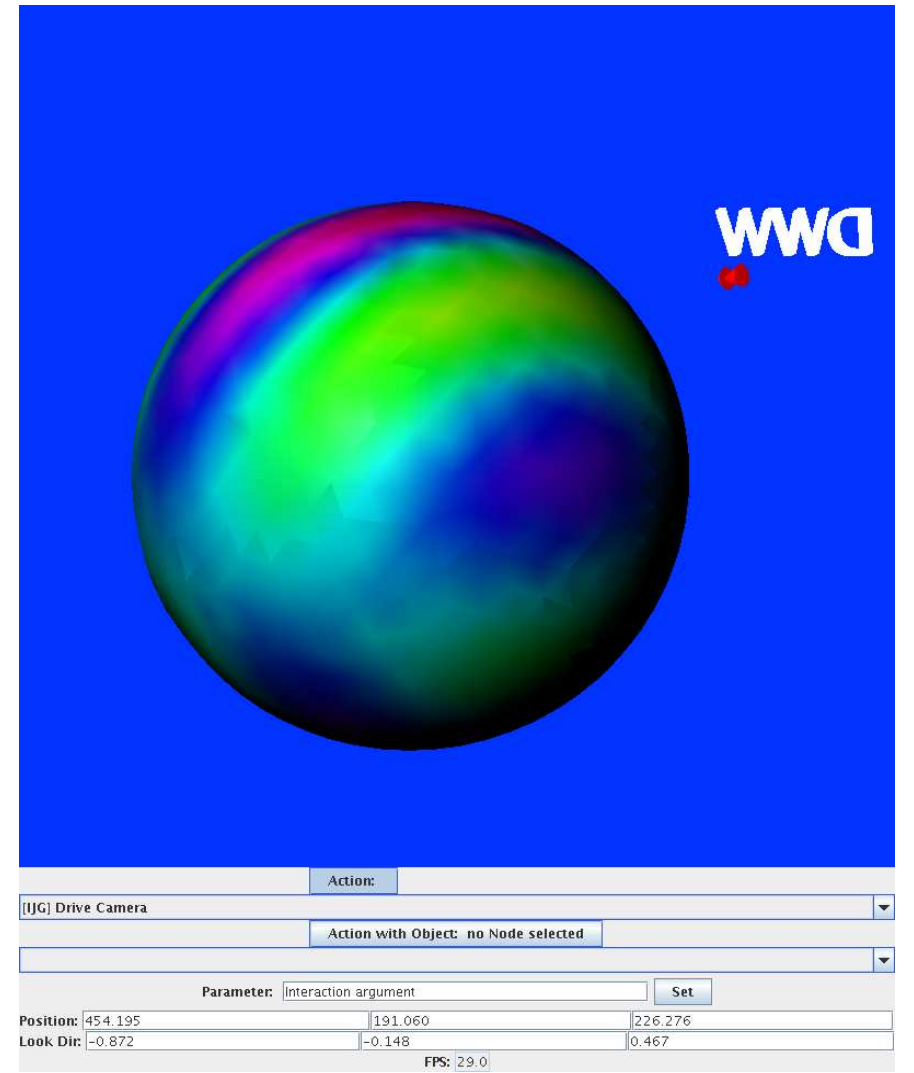

Fig. 3. A snapshot from a collaborative RAVE visualization session showing an avatar.

\section{Implementation Details}

The GECEM portal is based on GridSphere 2.0.4 and GridPortlets 1.1.1. GridSphere was deployed in the Tomcat 5.0.30 servlet container. The GECEM services for mesh generation and CEM solver migration are simply wrapped legacy executables. In each case, the original Fortran code was modified slightly to make it callable from $\mathrm{C}$, and the 
$\mathrm{C}$ code was wrapped as a Java program using the Java Native Interface. The resulting Java program was then deployed as a GT3.2 service [9].

Authentication of users of the GECEM resources is based on e-Science certificates. These are X.509 certificates issued by the UK e-Science certificate authority. Since the services accessed through the GECEM portal are based on GT3.2, the portal makes use of the Grid Security Infrastructure (GSI) for the authentication of users, services, and resources. GSI also provides for "single sign-on" to Grid resources and the delegation of credentials. The GECEM portal uses the MyProxy online credential repository [10] managed by the Grid Operations Support Centre of the UK e-Science programme. The MyProxy Upload Tool, developed in the CCLRC DataPortal project, is used to upload a user's proxy credentials to the MyProxy repository. GridFTP is used to perform secure file transfers between sites in the GECEM Grid.

Security is an important requirement in the GECEM project. OGSA allows a number of different authorization mechanisms. In GECEM, security is implemented through the gridmap mechanism. The gridmap file on a given machine contains an entry for each person who can access the grid resources on that machine. It consists of a list of users with Distinguished Name (DN) and user account name. The gridmap file is used to check the authorization of users - only users whose DN appears in the gridmap file can use that resource. For example, for a user to use the GECEM portal, the DN of the user must be defined in the gridmap file of each of the machines hosting the GECEM services so that the user can transfer files from the user's local machine to the services hosts. In addition, the gridmap mechanism is also used by the service hosts to check whether a user is authorized to invoke the corresponding service. Further details of the Grid Security Infrastructure used in GT3 can be found in [11].

\section{Related Work}

The GridSphere portal framework is used across a range of scientific disciplines to create portlet-based portal interfaces . For example, the e-Physics portal developed by researchers at The University of Melbourne has been used to perform parameter sweep studies for a magneto-hydrodynamics astrophysics code, ZeusMP [12]. Unlike the GECEM portal, where the user is responsible for selecting between semantically equivalent services, in the e-Physics portal resource selection is usually done automatically by the Gridbus Broker. This difference arises from the distinct modes of use for which the GECEM and e-Physics portals were designed. The GeneGrid portal [13] is another example of a scientific portal based on GridSphere, and provides access to a virtual bioinformatics laboratory that allows users to construct experiments by either composing new workflows or reusing workflows created previously. The Astrophysics Simulation Collaboratory [14] uses the GridSphere portal framework to manage numerical relativity simulations based on the Cactus Computational Toolkit [15].

The portals mentioned in the previous paragraph all provide end-user interfaces for particular application domains. The P-GRADE portal supports the composition and execution of workflows, and is not tied to any specific application domain [16]. The P-GRADE portal is similar in some respects to the GECEM portal: both portals are based on GridSphere, both represent the input/output of data to/from a workflow node in terms of files, and both make use of certificates, GSI, and MyProxy servers in the 
authorization and authentication of users and resources. The P-GRADE portal provides a workflow editor that may be used to create new workflows and edit existing ones.

There are a number of portal frameworks, in addition to GridSphere, that are intended to simplify the development of grid portals. GridPort [17], Sakai ${ }^{2}$, and uPortal ${ }^{3}$ are some examples of these frameworks. Of these, GridPort and GridSphere are specifically aimed at supporting the development of grid portals, while Sakai and uPortal are used for building campus portals for use at higher education institutions. Although GridSphere was developed for supporting Grid use, it also has been used widely as a portal framework in its own right to support web application development unrelated to the Grid. On the other hand, grid portal developments on uPortal also exist. For example, the Open Grid Computing Environment (OGCE) [18] has been developed using both the GridSphere and uPortal portlet containers. It should be noted that GridPort has now been absorbed within the OGCE project.

\section{Summary and Conclusions}

We have presented a grid-based web portal for computational electromagnatics based on a combination of GridSphere, GridPortlets, the GECEM portlets to support surface and volume mesh generation, CEM solver migration of simulation codes, and the RAVE portlet for collaborative visualization of the simulation output. The GECEM portal supports the composition of applications from service-based components, the execution and monitoring of such applications on remote resources, and collaborative visualization and exploration of the application results. The GECEM portlets used to select input data sets and services for GECEM jobs have been described. A UDDI registry is used to publish and discover the mesh generation and CEM migration services. The portlets approach to providing a better interface for scientists has been described, and OGSA-compliant grid services for mesh generation and CEM solver migration have been discussed. Future work will examine the archiving of data sets in a virtual data store, and migrating the portal to use "pure" web services based on emerging web service standards.

\section{Acknowledgements}

The authors would like to acknowledge the support of the U.K. Department of Trade and Industry, and many helpful discussions with their collaborators on the GECEM project, especially Yu Chen and Jason Jones of The University of Wales, Swansea who developed the surface mesh generation, volume mesh generation, and CEM solver migration services. It is also a pleasure to acknowledge the contributions of Ganesan Subramaniam and Ian Grimstead for their work in developing the RAVE portlet.

\section{References}

[1] D. Gannon, J. Alameda, O. Chipara, M. Christie, V. Dukle, L. Fang, M. Farrellee, G. Kandaswamy, D. Kodeboyina, S. Krishnan, C. Moad, M. Pierce, B. Plale, A. Rossi, Y. Simmhan, A. Sarangi,

\footnotetext{
2 http://www.sakaiproject.org/

3 http://www.uportal.org/
} 
A. Slominski, S. Shirasuna, T. Thomas, Building grid portal applications from a web service component architecture, IEEE Proceedings 93 (3) (2005) 551-563.

[2] I. Foster, C. Kesselman, J. M. Nick, S. Tuecke, The physiology of the grid: An open grid services architecture for distributed systems integration (June 2002).

URL http://www.globus.org/research/papers/ogsa.pdf

[3] D. W. Walker, J. P. Giddy, N. P. Weatherill, J. W. Jones, A. Gould, D. Rowse, M. Turner, GECEM: Grid-Enabled Computational Electromagnetics, in: Proceedings of the UK e-Science All Hands Meeting, 2003, pp. 436-443.

[4] L. Clement, A. Hateley, C. von Riegen, T. Rogers, UDDI version 3.0.2, http://uddi.org/pubs/uddi_v3.htm (2004).

[5] F. Bellas, Standards for second-generation portals, IEEE Internet Computing 8 (2) (2004) 54-60.

[6] J. Novotny, M. Russell, O. Wehrens, Gridsphere: A portal framework for building collaborations, Concurrency and Computation: Practice and Experience 16 (5) (2004) 503-513.

[7] M. Russell, O. Wehrens, J. Novotny, The gridportlets web application: A grid portal framework, in: Proceedings of the Sixth International Conference on Parallel Processing and Applied Mathematics, Springer, 2006, Lecture Notes in Computer Science, Volume 3911.

[8] I. J. Grimstead, N. J. Avis, D. W. Walker, R. N. Philp, Resource-aware visualization using web services, in: Proceedings of the UK e-Science All Hands Meeting, 2005.

[9] M. Lin, D. W. Walker, Y. Chen, J. W. Jones, A web service architecture for GECEM, in: S. J. Cox (Ed.), Proceedings of the UK e-Science All Hands Meeting, 2004.

[10] J. Novotny, S. Tuecke, V. Welch, An online credential repository for the grid: MyProxy, in: Proceedings of the Tenth International Symposium on High Performance Distributed Computing, IEEE Press, 2001, pp. 104-111.

[11] V. Welch, F. Siebenlist, I. Foster, J. Bresnahan, K. Czajkowski, J. Gawor, C. Kesselman, S. Meder, L. Pearlman, S. S. Tuecke, Security for grid services, in: Proceedings of the 12th IEEE International Symposium on High Performance Distributed Computing, 2003, pp. 48-57.

[12] B. Beeson, S. Melnikoff, S. Venugopal, D. G. Barnes, A portal for grid-enabled physics, in: R. Buyya, P. Coddington, P. Montague, R. Safavi-Naini, N. Sheppard, A. Wendelborn (Eds.), Proceedings of the 2005 Australasian Workshop on Grid Computing and e-Research (AusGrid 2005), ACM Press, 2005, pp. 13-20.

[13] N. Kelly, P. Jithesh, S. Wasnik, R. McLaughlin, F. Fragoso, P. Donachy, T. Harmer, R. Perrott, M. McCurley, M. T. nand J. Johnston, S. McKee, The genegrid portal: A user interface for a virtual bioinformatics laboratory, in: Proceedings of the UK e-Science All Hands Meeting, 2005.

[14] R. Bondarescu, G. Allen, G. Daues, I. Kelley, M. Russell, E. Seidel, J. Shalf, M. Tobias, The astrophysics simulation collaboratory portal: A framework for effective distributed research, Future Generation Computer Systems 21 (2) (2005) 259-270.

[15] T. Goodale, G. Allen, G. Lanfermann, J. Masso, T. Radke, E. Seidel, J. Shalf, The cactus framework and toolkit: Design and applications, in: Proceedings of the Fifth International Conference on Vector and Parallel Processing, Springer, 2003.

[16] P. Kacsuk, G. Sipos, Multi-grid, multi-user workflows in the p-grade grid portal, Journal of Grid Computing 3 (3-4) (2005) 231-238.

[17] M. Thomas, J. Boisseau, Building grid computing portals: The NPACI grid portal toolkit, in: F. Berman, G. Fox, T. Hey (Eds.), Grid Computing: Making the Global Infrastructure a Reality, J. Wiley and Sons, Chichester, 2003.

[18] J. Alameda, M. Christie, G. Fox, J. Futrelle, D. Gannon, M. Hategan, G. Kandaswamy, G. von Laszewski, M. A. Nacar, M. Pierce, E. Roberts, C. Severance, M. Thomas, The Open Grid Computing Environments collaboration: Portlets and services for science gateways, Concurrency and Computation: Practice and Experience, to appear. 